\title{
Estratégias de Ensino Ativas e Desenvolvimento de Competências de Estudantes de Administração: proposta de uma escala de mensuração
}

\author{
Silva, Anielson Barbosa da ${ }^{a}$, Dias Júnior, José Jorge Lima ${ }^{b}$, Canós-Darós, Lourdes \\ ${ }^{a}$ Universidade Federal da Paraíba - UFPB, Brasil, anielson @ uol.com.br, ${ }^{\text {b }}$ Universidade Federal da \\ Paraíba - UFPB, Brasil, jorge@dce.ufpb.br c Universitat Politècnica de València- UPV, \\ loucada@omp.upv.es
}

\begin{abstract}
Resumo
Este artigo objetiva propor uma escala de mensuração para avaliar o uso de estratégias de ensino ativas no desenvolvimento de competências de estudantes de Administração. A pesquisa, de natureza quantitativa $e$ descritiva, foi realizada com uma amostra de 369 alunos do curso de Administração da Universidade Federal da Paraíba, Brasil. A partir da utilização de métodos multivariados de análise, foi possível validar a escala de mensuração com cinco construtos (Consciência sobre a prática gerencial, Trabalho em equipe, Consciência social e emocional, Planejamento e resolução de problemas e Visão sistêmica) e 23 itens. Além disso, foi possível identificar que a estratégia de ensino de aprendizagem baseada em problemas foi a que mais contribuiu no desenvolvimento das competências dos estudantes.
\end{abstract}

Palavras-chave: estratégias de ensino, competências, estudantes, administração, escala de mensuração.

\section{Introdução}

O ambiente de formação na educação superior demanda dos agentes responsáveis pelo planejamento acadêmico o estabelecimento de métodos de ensino mais inovadores e orientados para a ação. Os alunos precisam ser convidados a viverem experiências significativas por meio do estabelecimento de um ambiente de aprendizagem em que as dimensões físicas, sociais, emocionais e tecnológicas (Silva, Silva \& Coelho, 2019) devem estar articuladas com o mundo do trabalho.

Wlodkowski (2004) destaca a importância de criar ambientes de aprendizagem motivadores 
para que os estudantes sejam mais efetivos na construção de significados a partir da informação e da experiência. Em um ambiente de aprendizagem em que há um propósito e uma tarefa desafiadora, geralmente o aluno se sente motivado a aprender. Entretanto, o autor também indica que a motivação para aprender pode desaparecer em um milésimo de segundo quando fatores associados ao ambiente (sala quente, baixo desempenho no teste, discussão chata), além de fatores culturais que levam as pessoas a terem diferentes necessidades e perspectivas, tornam a atividade de ensino desafiadora.

Rinaudo, Chiecher e Donolo (2003) ressaltam que várias discussões teóricas e empíricas enfatizaram a importância de atender não apenas a dimensão cognitiva da aprendizagem, mas também os aspectos afetivos e motivacionais que estimulem os alunos a participarem de forma ativa e tornar os processos de aprendizagem mais significativos.

A utilização de estratégias de ensino ativas podem contribuir para o desenvolvimento de competências profissionais e promover o engajamento dos alunos, motivando-os a agir individualmente, em pares ou em pequenos grupos em discussões que sejam significativas e difundidas no ambiente da sala de aula (Wurdinger \& Carlson, 2010).

Silva (2016) ressalta que o uso de estratégias ativas de aprendizagem aproxima o professor e os alunos, além de promover uma maior integração entre a teoria e a prática, a reflexão e a ação. Nesse sentido, Jennings (1996) destaca a efetividade do método de caso no desenvolvimento de habilidades de comunicação, habilidades interpessoais e de integração que são relevantes para a prática de gestão e o pensamento estratégico.

Outros estudos revelaram, em seus resultados, a contribuição do uso de estratégias ativas de ensino na aprendizagem e no desenvolvimento de competências de estudantes, tais como aprendizagem baseada em problemas (Silva, Bispo, Rodriguez \& Vasquez, 2018), casos para ensino (Alberton \& Silva, 2018), filmes (Schultz \& Quinn, 2013) e história em quadrinhos (Silva, Santos \& Bispo, 2017; Brandão \& Silva, 2018).

Este artigo objetiva propor uma escala de mensuração para avaliar o uso de estratégias de ensino ativas no desenvolvimento de competências de estudantes de Administração. Além da proposta da escala, identificamos a contribuição de quatro estratégias ativas de aprendizagem (aprendizagem baseada em problemas, método de casos para ensino, filmes e história em quadrinhos) no desenvolvimento das competências identificadas na escala.

As principais contribuições do artigo são: (a) propor uma escala para avaliação da contribuição das estratégias de ensino ativas no desenvolvimento de competências dos estudantes; (b) auxiliar na identificação de competências para o planejamento de programas e cursos de desenvolvimento gerencial; e (c) reforçar a importância do uso de estratégias de ensino ativas para tornar o processo de aprendizagem mais significativo para os estudantes. 


\section{Método}

A pesquisa teve como objetivo propor escala de mensuração para avaliar o uso de estratégias de ensino ativas no desenvolvimento de competências de estudantes de Administração. Para alcançar o objetivo, foi realizado um estudo quantitativo com estudantes do curso de Administração da Universidade Federal da Paraíba (UFPB), Brasil.

\subsection{Coleta de dados}

A coleta de dados foi realizada nos anos de 2016 e 2017 e ocorreu após a utilização das estratégias de ensino ativas pelos professores do curso de graduação em Administração da UFPB das disciplinas de Gestão do Conhecimento nas Organizações, Processo Decisório e Desenvolvimento Gerencial, Comportamento do Consumidor, Sistema de Informação e Decisão, Administração de Recursos Humanos.

O instrumento de coleta foi um questionário estruturado em duas partes. A primeira parte, com 29 questões, foi adaptada do estudo realizado por Pereira (2012) para avaliar os fatores determinantes para utilização de casos para ensino na percepção de alunos e professores. Para captar a percepção do aluno sobre a utilização de uma das estratégias ativas de ensino pelo professor (aprendizagem baseada em problemas, método de casos para ensino, filmes e história em quadrinhos), foi utilizada uma escala intervalar com 11 pontos ( 0 a 10$)$. Quanto mais próximo de 0 o aluno indicasse a sua resposta, menor a contribuição da estratégia de ensino ativa na variável analisada e, quanto mais próximo de 10 se posicionasse, maior a contribuição da estratégia de ensino ativa na variável analisada. Por fim, a última parte do instrumento, com 7 questões, teve como objetivo caracterizar o perfil dos alunos que participaram do estudo.

\subsection{Caracterização da Amostra e Análise de Dados}

Após a coleta, foi realizada uma limpeza e posterior retirada dos dados faltantes. Desse modo, a base de dados foi composta por 371 alunos de graduação do curso de Administração, dos quais $81 \%$ já cursaram mais de 4 semestres, 52,6\% estudam no turno matutino, 70,2\% iniciaram o curso até 2013 e 43,6\% trabalham com vínculo empregatício. A média e a mediana da idade dos alunos é de 25 anos e 23 anos, respectivamente, variando de 18 a 56 anos. Em relação ao sexo, $50 \%$ são homens e $50 \%$ são mulheres. O processo de análise dos dados envolveu uma análise estatística descritiva do perfil dos respondentes, análise fatorial exploratória (AFE) para identificação dos construtos e análise fatorial confirmatória (AFC) para validação da escala. Após o processo de validação da escala, foi realizada uma análise 
Estratégias de Ensino Ativas e Desenvolvimento de Competências de Estudantes de Administração: proposta de uma escala de mensuração

de variância (anova) para analisar a contribuição das estratégias ativas de aprendizagem.

\section{Resultados}

A primeira etapa foi submeter os itens à técnica de AFE para verificar quais construtos emergiriam. O método de rotação adotado foi o varimax, que aplica uma rotação ortogonal visando maximizar as cargas fatoriais da matriz fatorial (Hair, Black, Babin, Anderson et al., 2009). Sobre o número de fatores, adotou-se a forma livre para que a técnica recomende a quantidade de fatores, utilizando o critério Kaiser, em que se seleciona a quantidade de fatores com os autovalores maiores que um (Hair, Black, Babin, Anderson et al., 2009).

Aoexecutar a AFE com 29 itens, emergiram 7 fatores subjacentes. Foram removidos os itens com comunalidades abaixo de 0,4 e cargas fatoriais abaixo de 0,5. Depois dos ajustes, 23 itens foram submetidos novamente à $\mathrm{AFE}$, por meio da qual 5 fatores foram sugeridos. A confiabilidade, por meio do alpha de Cronbach, apresentou índices satisfatórios $(\propto \geq 0,7)$. Com o resultado, discutiu-se se os itens faziam sentido teórico em cada um dos seus fatores. Cada um desses foi denominado considerando o alinhamento teórico de seus itens. Portanto, os 5 fatores que emergiram são: Consciência sobre a prática gerencial (6 itens), Trabalho em equipe (4 itens), Consciência social e emocional (6 itens), Planejamento e resolução de problemas (4 itens), e Visão sistêmica (3 itens).

Em seguida, partiu-se para a aplicação da AFC com o objetivo de avaliar a qualidade de ajustamento do modelo de medida teórico à estrutura correlacional observada entre os itens da escala. Nesse caso, o número de fatores é estabelecido a priori de acordo com a teoria. Considerando que o resultado da AFE demonstrou um bom alinhamento teórico, foi utilizada essa estrutura para ser confirmada via AFC.

O resultado da AFC demonstrou uma boa qualidade no ajuste $\left(\chi^{2}=692,33 ; \mathrm{gl}=213\right.$; TLI=0,93; CFI=0,94; RFI=0,91; IFI=0,94; RMSEA=0,09; SRMR=0,06), dentro de índices de referência normalmente utilizados na literatura (Hair, Black, Babin, Anderson et al., 2009). Além disso, foi analisada a confiabilidade por meio do alpha de Cronbach, Confiabilidade Composta (CC) e Average Variance Extracted (AVE). As referências são, respectivamente, 0,7, 0,7 e 0,5. A Tabela 1 apresenta os resultados de cada um dos fatores. Apenas o fator 'Consciência social e emocional' ficou abaixo da referência na AVE, mas bastante próximo dela. Considerando a relevância dos seus itens, decidiu-se mantê-los. A Tabela também apresenta as correlações entre os construtos. 
Tabela 1. Índices de confiabilidade dos fatores analisados via AFC

\begin{tabular}{lccccc}
\hline \multicolumn{1}{c}{ Construtos } & 1 & 2 & 3 & 4 & 5 \\
\hline 1. Consciência sobre a prática gerencial & 1 & & & & \\
2. Trabalho em equipe & 0,554 & 1 & & & \\
3. Consciência social e emocional & 0,706 & 0,813 & 1 & & \\
4. Planejamento e resolução de problemas & 0,781 & 0,716 & 0,723 & 1 & \\
5. Visão sistêmica & 0,818 & 0,579 & 0,664 & 0,754 & 1 \\
\hline Alpha & 0,893 & 0,865 & 0,834 & 0,842 & 0,759 \\
CC & 0,838 & 0,768 & 0,786 & 0,781 & 0,734 \\
AVE & 0,568 & 0,581 & 0,452 & 0,565 & 0,549 \\
\hline
\end{tabular}

Fonte: Dados da pesquisa (2019)

Também foram verificados que os escores padronizados atingiram índices maiores que 0,6 , e que suas respectivas significâncias apresentaram $p<0,05$. Nesse sentido, a validade estrutural da escala foi validada, indicando sua confiabilidade. A ferramenta utilizada para executar as técnicas foi a linguagem $\mathrm{R}$, juntamente com pacotes específicos como o lavaan.

A partir da validação da escala, foi possível avaliar a contribuição de quatro estratégias de ensino ativas no desenvolvimento de competências. A avaliação foi possível porque o questionário tinha como objetivo avaliar a utilização de estratégias de ensino ativas, como aprendizagem baseada em problemas, casos para ensino, filmes e histórias em quadrinhos pelo professor em sala de aula. Para cada atributo associado aos cinco construtos validados na escala, o aluno teria que indicar, em uma escala de 0 a 10, o nível de contribuição da estratégia de ensino no desenvolvimento da competência.

A Tabela 2, a seguir, apresenta as médias obtidas em cada uma das competências por estratégia de ensino. Foi realizada uma análise de variância (anova) para verificar se havia diferenças significativas entre as médias dos grupos. Como mostra a Tabela 2, houve diferença significativa, quando consideramos o p-valor menor do que 0,05 , nas médias das competências consciência sobre a prática gerencial, planejamento e resolução de problemas e visão sistêmica. 
Estratégias de Ensino Ativas e Desenvolvimento de Competências de Estudantes de Administração: proposta de uma escala de mensuração

Tabela 2: Contribuição das estratégias de ensino ativas no desenvolvimento das competências

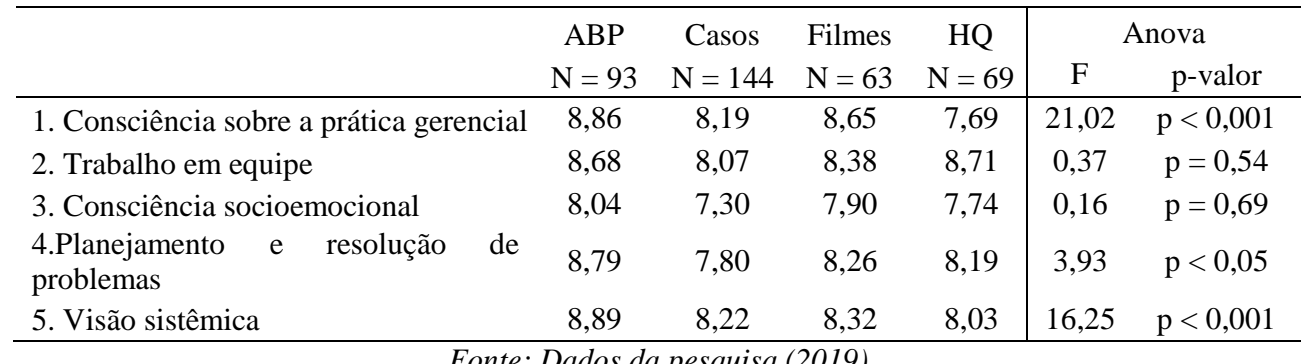

Fonte: Dados da pesquisa (2019)

Os resultados indicam que a estratégia de aprendizagem baseada em problemas foi a que mais contribuiu no desenvolvimento das competências de consciência sobre a prática gerencial, de planejamento e resolução de problemas e de visão sistêmica. Para as competências de trabalho em equipe e de consciência socioemocional, os resultados indicam bons níveis de contribuição de todas as estratégias de ensino, tomando como referência a média.

\section{Considerações Finais}

Este estudo teve como objetivo propor uma escala de mensuração para avaliar o uso de estratégias de ensino ativas no desenvolvimento de competências de estudantes de Administração. Os resultados do processo de validação possibilitaram a identificação de cinco construtos, aqui definidos como competências, e 23 atributos associados a elas. A Tabela 3, a seguir, apresenta uma definição de cada competência e os atributos associados. 
Tabela 3: Competências desenvolvidas a partir da utilização de estratégias ativas de ensino.

\begin{tabular}{|c|c|}
\hline Competência & Descritores \\
\hline $\begin{array}{l}\text { Consciência sobre a prática gerencial: } \\
\text { capacidade de aprender sobre a ação } \\
\text { gerencial, por meio da percepção da } \\
\text { relação entre teoria e prática, ampliando o } \\
\text { conhecimento das atividades e do } \\
\text { funcionamento de uma empresa. }\end{array}$ & $\begin{array}{l}\text { - Aprendizado sobre a ação gerencial. } \\
\text { - Associação da teoria à prática gerencial. } \\
\text { - Visão do gestor sobre o funcionamento da empresa. } \\
\text { - Conhecimento de atividades pertinentes à prática } \\
\text { profissional. } \\
\text { - Novos conhecimentos sobre o funcionamento da } \\
\text { empresa. }\end{array}$ \\
\hline $\begin{array}{l}\text { Trabalho em equipe: capacidade de } \\
\text { interação com os colegas visando à troca } \\
\text { de informações, à resolução de problemas } \\
\text { e à melhoria da motivação para trabalhar } \\
\text { em equipe. }\end{array}$ & $\begin{array}{l}\text { - Capacidade de comunicação com os seus colegas. } \\
\text { - Habilidade de fornecer informações para os colegas. } \\
\text { - Eficácia na resolução de problemas em grupo. } \\
\text { - Motivação para trabalhar em equipe. }\end{array}$ \\
\hline $\begin{array}{l}\text { Consciência socioemocional: capacidade } \\
\text { de compreender suas atitudes e a dos } \\
\text { colegas para lidar com a insegurança, } \\
\text { resolver conflitos interpessoais, trabalhar } \\
\text { de forma independente, por meio da } \\
\text { exploração de comportamentos } \\
\text { individuais. }\end{array}$ & $\begin{array}{l}\text { - Consciência sobre as suas atitudes administrativas. } \\
\text { - Consciência sobre as atitudes dos colegas. } \\
\text { - Capacidade de lidar com a insegurança. } \\
\text { - Capacidade de resolver conflitos interpessoais. } \\
\text { - Exploração de um comportamento que conhecia, } \\
\text { mas ainda não havia vivenciado. } \\
\text { - Habilidade de trabalhar independentemente. }\end{array}$ \\
\hline $\begin{array}{l}\text { Planejamento e resolução de problemas: } \\
\text { capacidade de resolver problemas a partir } \\
\text { do planejamento de operações do negócio, } \\
\text { implantação de ideias e planos, utilizando } \\
\text { o pensamento reflexivo. }\end{array}$ & $\begin{array}{l}\text { - Habilidade para resolver problemas práticos. } \\
\text { - Capacidade de planejamento das operações de } \\
\text { negócios. } \\
\text { - Capacidade para implementar suas próprias ideias e } \\
\text { planos. } \\
\text { - Pensamento reflexivo. }\end{array}$ \\
\hline $\begin{array}{l}\text { Visão sistêmica: capacidade de aprender a } \\
\text { integrar as diversas áreas funcionais de } \\
\text { uma empresa para identificar e analisar } \\
\text { problemas a partir de diferentes } \\
\text { perspectivas identificadas na interação } \\
\text { com a equipe. }\end{array}$ & $\begin{array}{l}\text { - Integração da aprendizagem em diversas áreas: } \\
\text { (Contabilidade, Finanças, Marketing, etc.). } \\
\text { - Capacidade de identificar os problemas gerenciais. } \\
\text { - Análise de um problema a partir de diferentes } \\
\text { pontos de vista na discussão do grupo. }\end{array}$ \\
\hline
\end{tabular}

Fonte: Dados da pesquisa (2019)

A escala proposta pode auxiliar na delimitação das competências que podem ser desenvolvidas em programas de formação, tanto em nível de graduação como de pósgraduação, uma vez que indica atributos associados ao contexto da prática gerencial. Garvin (2007) identifica algumas metas de programas de desenvolvimento gerencial e destaca acapacidade de levar os estudantes a compreenderem o negócio e adquirirem conhecimento sobre a empresa, além de ajudá-los a lidar com as pressões para a resolução de problemas e melhorar o seu desempenho no trabalho. Os resultados deste estudo 
reforçam a relevância das competências propostas para a estruturação de programas de aprendizagem gerencial, por meio de ações que articulem a educação e o desenvolvimento gerencial, como preconizam Fox (1997) e Mintzberg (2006).

Os estudos realizados por Jennings (2000), Chang (2003) e Chang, Jennings, To e Sun (2005) para avaliar o uso de três estratégias de ensino (projetos de consultoria, simulação e método de caso) possibilitaram identificar que a utilização das estratégias promove uma experiência de aprendizagem mais significativa, sobretudo a simulação de novos negócios.

Uma das implicações práticas da escala de avaliação do desenvolvimento de competências a partir da utilização de estratégias de ensino ativas é a identificação da contribuição das estratégias de ensino utilizadas pelo professor na aprendizagem dos alunos. Este estudo revelou que a aprendizagem baseada em problemas teve a maior contribuição no desenvolvimento de três das cinco competências da escala validada, reforçando os resultados de Silva, Bispo, Rodriguez \& Vasquez (2018).

Esse achado reforça o posicionamento de Jennings (2002) de que os métodos de ensino podem ter contribuições diferentes na aprendizagem dos estudantes. Por outro lado, a utilização de estratégias de ensino diferentes no processo de aprendizagem pode potencializar o desenvolvimento de competências diferentes. Assim, o mais importante é o resultado final alcançado por meio da participação no curso.

\section{Referências}

Alberton, A., \& Silva, A. B. (2018). Como Escrever um Bom Caso para Ensino? Reflexões sobre o Método. RAC - Revista de Administração Contemporânea, 22, 745-761.

Brandão, J.M.F., \& Silva, A. B. (2018) . Dejando Volar la Imaginación: el Uso de la Historieta como Estrategia de Enseñanza en Hotelería. Estudios y Perspectivas en Turismo, 27, 233-255.

Chang, J. (2003). Strategic Management: an Evaluation of the Use of Three Learning Methods in Hong Kong. Developments in Business Simulation \& Experiential Learning, 30, 146-151.

Chang, J., Jennings, D., To, C., \& Sun, L. (2005). Strategic Management: an Evaluation of the Use of Three Learning Methods in China. Developments in Business Simulation \& Experiential Exercises, $32,76-78$.

Fox, S. (1997). From Management Education and Development to the Study of Management Learning. In: Burgoyne, J., \& Reynolds, M. Management Learning: Integrating Perspectives in Theory and Practice. London: Sage Publications.

Garvin, D. A. (2007). Teaching Executives and Teaching MBA's: Reflections on the Case Method. Academy of Management Learning \& Education, 6(3), 364-374. 
Hair, J. F., Black, W. C., Babin, B. J., Anderson, R. E., \& Tatham, R. L. (2009). Análise Multivariada de Dados. Porto Alegre: Bookman Editora.

Jennings, D. (1996). Strategic Management and the Case Method. Journal of Management Development, 15(9), 4-12.

Jennings, D. (2000). Strategic Management: an Evaluation of the Use of Three Learning Methods.

Development in Business Simulation \& Experiential Learning, 27, 20-25.

Jennings, D. (2002) Strategic Management: an Evaluation of the Use of Three Learning Methods. The Journal of Management Development, 21(9/10), 655-665.

Mintzberg, H. (2006). MBA: Não Obrigado - Uma Visão Crítica sobre a Gestão e o Desenvolvimento de Gerentes. Porto Alegre: Bookman.

Pereira, L. S. (2012). Método do Caso para o Ensino: Fatores Determinantes para sua Utilização na Visão de Alunos e Professores. Dissertação de Mestrado, Universidade do Vale do Itajaí, Itajaí, Santa Catarina, Brasil.

Rinaudo, M. C., Chiecher, A., \& Donolo, D. (jun. 2003). Motivación y Uso de Estrategias en Estudiantes Universitarios. Su Evaluación a partir del Motivated Strategies Learning Questionnaire, Anales de Psicología, 19(1), 107-119.

Schultz, P. L., \& Quinn, A. S. (2013). Lights, Camera, Action! Learning About Management With StudentProduced Video Assignments. Journal of Management Education, 38(2), 234-258.

Silva, A. B., Bispo, A. C. K. A, Rodriguez, D. G., \& Vasquez, F. I. F. (2018). Problem-Based Learning: a Proposal for Structuring PBL and its Implications for Learning among Students in an Undergraduate Management Degree Program. REGE - Revista de Gestão, 25, 160-177.

Silva, A. B., Santos, G. T., \& Bispo, A. C. K. A. (2017).The Comics as Teaching Strategy in Learning of Students in an Undergraduate Management Program. RAM - Revista de Administração Mackenzie, 18, 40-65.

Silva, A. B. (2016). Action Learning: Lecturers, Learners, and Managers at the Center of Management Education. In M. T. Lepeley, E. V. Kimakovitz, R. Bardy. (Eds.). Human Centered Management in Executive Education: Global Imperatives, Innovation and New Directions (1 ed.) (pp. 126-139). London: Palgrave Macmillan.

Silva, M. D. S., Silva, A. B., \& Coelho, A. L. A. L. (2019). Implications of the Learning Environment in a Professional Master's Degree in Business Administration in Brazil. Learning Environments Research, 22, 1-20.

Wlodkowski, R.J. (2004). Creating Motivating Learning Environments. In: M. W. Galbraith (Ed.) Adult Learning Methods: a Guide for Effective Instruction (3. ed) (pp. 141-164). Florida: Krieger 
Estratégias de Ensino Ativas e Desenvolvimento de Competências de Estudantes de Administração: proposta de uma escala de mensuração

Publishing Company.

Wurdinger, S. D. \& Carlson, J. A. (2010). Teaching for Experiential Learning: Five Approaches That Work. United Kingdom: Rowman \& Littlefield Education. 